\title{
Treatment of Bilateral Vocal Fold Immobility
}

\author{
Joseph P. Bradley $•$ Adam M. Klein
}

Published online: 19 February 2014

(C) Springer Science+Business Media New York 2014

\begin{abstract}
The purpose of this review is to analyze the literature updates on bilateral vocal fold immobility (BVFI) from 2011 to 2013. Whereas management of BVFI has not changed substantially in recent years, some modifications to techniques have been reported in the literature since 2011. These will be discussed in detail. Management of BVFI continues to challenge the laryngeal surgeon, especially with regard to voice preservation while establishing an adequate airway. Although static procedures continue to be the mainstay of treatment, focus is turning toward the development of new devices that would enable dynamic restoration of movement.
\end{abstract}

Keywords Bilateral vocal fold paralysis - Bilateral vocal fold immobility $\cdot$ Posterior glottic stenosis

\section{Introduction}

Bilateral vocal fold immobility (BVFI) is a complicated medical condition that can have profound effects on patients. Irrespective of etiology, presentation can range from mild dyspnea to respiratory distress and severe biphasic stridor. Even for providers accustomed to dealing with difficult airways, treating a patient with this condition can be a harrowing experience. Unlike patients with unilateral vocal fold immobility, there is, typically, no breathy

J. P. Bradley $(\bowtie) \cdot$ A. M. Klein $(\bowtie)$

Emory Voice Center, Department of Otolaryngology-Head and

Neck Surgery, Emory School of Medicine, 550 Peachtree St NE, 9th Floor, Suite 4400, Atlanta, GA 30308, USA

e-mail: joseph.bradley@emory.edu

A. M. Klein

e-mail: aklein4@emory.edu dysphonia, given that the vocal folds are more tightly approximated in the bilateral condition. Patients may have some vocal limitations, however, because of poor breathing support or vocal fold adduction or paramedian positioning of the vocal folds.

BVFI may develop from several etiologies, which are broadly categorized into cricoarytenoid (CA) joint fixation and paralysis. The complicated and lengthy pathway of the recurrent laryngeal nerves (RLN) during embryologic development leaves them vulnerable to several causes of injury. Bilateral vocal fold paralysis is more commonly associated with iatrogenic causes-the most common being surgery $(26-59 \%)$ and intubation $(1-31 \%)[1 \bullet \cdot]$. Other known causes include external beam radiation, neoplasia, congenital neurologic causes (Arnold-Chiari malformations, myelomeningoceles), diabetes mellitus, and other neuropathies (amyotrophic lateral sclerosis, cerebrovascular accident, myasthenia gravis, etc.). CA joint fixation is most commonly associated with posterior glottis stenosis (PGS) and scarring. It may, however, be associated with other pathologies that affect the CA joint, including rheumatoid arthritis, systemic lupus erythematosis, gout, ankylosing spondylitis, Crohn's disease, and mumps.

\section{Surgical Techniques}

Most patients with BVFI will require intervention at some point; $\sim 4-14 \%$ of patients manage to avoid surgical treatment $[1 \bullet \cdot$. Nonsurgical options include respiratory retraining and botulinum toxin injections into the thyroarytenoid and lateral CA muscles. When contemplating the different types of surgical option currently available, it is important to review the major functions of the larynx, 
namely respiration, deglutition, and phonation, and to consider the effect on each treatment modality on each function. Intervention generally falls into three main classes: bypass of the obstruction, static opening of the airway, and dynamic opening of the airway.

\section{Bypass of the Obstruction}

The mainstay of therapy, and the most time-tested procedure, is the tracheotomy. It enables direct bypassing of the airway obstruction and is a technique in which essentially all otolaryngologists are proficient. However, it is associated with potential complications [2,3], the need for longterm care, and reduced quality of life [4]. For this reason, other management techniques have been refined over the past century, although tracheotomy may still be required before implementation of these other methods. Very little has changed with regard to tracheotomy as a management tool for treatment of BVFI.

\section{Static Opening}

\section{Endoscopic Options}

Review of the available literature between 2011 and 2013 reveals there have been two advances in endoscopic management of BVFI.

The endoscopic total arytenoidectomy introduced by Ossoff et al. [5] and the transverse posterior cordotomy introduced by Dennis and Kashima [6] have been the mainstays of endoscopic treatment of BVFI. Variations have been described by numerous authors over the years. Yilmaz [7•], from Turkey, recently described a modification of the endoscopic total arytenoidectomy which involves creation of a mucosal advancement flap and repositioning of the true vocal fold to widen the glottic aperture. In his technique, he removes an anteriorly-based triangular area of mucosa to gain access the arytenoid. He sharply dissects this away from its muscular attachments and separates it from the posterior vocal fold. He carefully preserves the mucosa medial to the arytenoid and creates a releasing incision immediately posterior to the membranous vocal fold extending inferiorly toward the subglottis. The free edge of the flap is then sutured posterolaterally near where the muscular process previously existed. Tension and lateralization is then applied to the vocal fold by taking its now-free posterior edge and suturing it posterolaterally immediately medial to the placement of the mucosal advancement flap. The author performed this technique on fifty patients during a five-year period. Four of these patients $(8 \%)$ required surgery more than once to become symptom-free or to be decannulated. Ten of the patients had a tracheotomy preoperatively, and all were decannulated within one month of surgery. Three patients who did not have a tracheotomy preoperatively required one postoperatively secondary to edema, but all three patients were decannulated one month after surgery. Voice handicap index (VHI-30) scores increased significantly, indicating a transition from mild to moderate voice handicap. There was no statistically significant change in functional outcome swallowing scale, indicating aspiration was not increased as a result of the procedure. The study did not have a control group with which to make direct comparisons between the techniques. Moreover, as the author indicates, although this procedure seems to work well for bilateral vocal fold paralysis, there is no evidence to indicate it would work for a scarred posterior larynx in the setting of PGS.

The second endoscopic technique introduced in the last two years is that reported by Semmler et al. [8•] for treatment of early stage PGS (Bogdasarian \& Olson Grade I or II). In this technique, the area of fibrosis and scarring is first resected endoscopically. The posterior glottic scar tissue is divided in the sagittal plane, with care being taken not to extend the incision back into the post-cricoid mucosa. A transverse incision is then made at the level of the inferiormost part of the arytenoid and extended anteriorly along the aryepiglottic fold. A more inferior incision $4 \mathrm{~mm}$ below the first is then made anterolaterally and the two are connected anteriorly by a vertical incision. This posteromedially-based mucosal advancement flap can then be rotated to cover the raw defect of the posterior glottis. The authors then used a fibrin glue to position the flap. This technique was performed on four patients, for three of whom the graft was completely successful and airway patency subsequently improved. The fourth patient had improvement of voice but no improvement in airway. The benefit of this technique is that it utilizes endolaryngeal mucosa away from the posterior larynx and so does not serve as a new locus for scarring. This study is limited by its sample size and its use for patients with lower grade PGS.

\section{Open Options}

Another technique is endoscopic suture laterofixation of the vocal fold to open the glottic airway. This technique can be performed alone or in conjunction with other intralaryngeal procedures (e.g. arytenoidectomy, submucous cordectomy). The technique, as classified by Lichtenberger, can be conducted in three different ways: reversible endoextralaryngeal lateralization, endoextralaryngeal laryngomicrosurgical lateralization, and endoextralaryngeal laryngomicrosurgical lateralization with arytenoidectomy [9], which are performed for cases of temporary immobility, cases without ankylosis of the CA joint, and cases involving ankylosis, respectively. 
Katilmiş et al. [10•] published their modification to the technique in 2011. They abandoned the completely endoscopic approach and added an open thyroplasty approach. They noted that some patients, after laterofixation, developed prolapse of the flaccid membranous vocal fold during inspiration. In this technique a thyroplasty window measuring $6-7 \mathrm{~mm}$ by $13-15 \mathrm{~mm}$ was situated $5 \mathrm{~mm}$ lateral from the midline and $5 \mathrm{~mm}$ superior to the inferior edge of the cartilage. This window was meant to incorporate both the glottic and ventricular folds. Two holes were drilled superior to the window and another two were drilled inferior to the window. The posterior-based holes were situated at the posterior corners of the window whereas the anterior-based holes were situated $5 \mathrm{~mm}$ behind the anterior-most edge of the window. Under direct laryngoscopic view, a 1-0 Prolene suture was passed internally from the anteroinferior hole and then passed externally through the posterosuperior hole. The suture was then passed into the posteroinferior hole and, endoscopically, passed externally through the anterosuperior hole to create a crossing of the suture internally and to lateralize both the true and false vocal folds. The crossing was endoscopically manipulated to keep the suture anterior to the vocal process of the arytenoid. After the suture had been tied-off externally, the cartilage window was replaced. This procedure was performed on five patients, and decannulation was successful for all four who had had a tracheotomy preoperatively. A follow-up paper by the same group reports study of seventeen patients who had the procedure then at least one year of follow-up [11•]. Three of the 17 patients had a tracheotomy preoperatively and all three were decannulated. The other 14 patients were all successfully extubated at the completion of the procedure. All patients experienced significant improvement $(P=0.0001)$ of their dyspnea on the Medical Research Council (MRC) dyspnea scale. When the patients subjectively measured their voice quality, by use of a five-point Likert scale, no significant difference was reported $(P=0.642)$. This study, while excellent in demonstrating a technique that can be easily performed without necessitating a tracheotomy, is also limited by its small sample size, by being a retrospective review, and the lack of a validated measure of voice outcome.

Suture lateralization is also associated with tearing, untying, or breaking of the suture, with gradual "cutting" of the suture into the underlying tissue. As a result of these complications with subsequent restenosis, many surgeons will perform tissue resection during revision. For this reason, Yagudin et al. [12•] created a novel technique, called the plastic cordotomy, to reshape the mucosal surfaces and change the airway shape without having to resect tissue. The idea was to create two transposing triangular-shaped flaps from the vocal fold and then unfold them in such a way as to create a concave surface. The authors performed this with a standard laryngofissure approach and a tracheotomy. The first incision is from the tip of the vocal process to the anterior commissure along the free edge of the vocal fold. The second incision is then made at $90^{\circ}$ to the first incision at its posterior-most edge and extended inferiorly into the subglottis. The tip of the vocal process is submucosally resected and a lateral portion of the thyroarytenoid muscle is also removed. The third and final incision is then made laterally from the anterior commissure to the lateralmost point of the posterior one-third of the ventricle. The flaps are then transposed and sutured into place, pulling the tissue laterally. The larynx was packed with gauze packingstrips and the laryngofissure was closed. A prospective study of this technique was performed for 21 consecutive female patients. Significant improvement of peak inspiratory flow and peak expiratory flow was observed, and no patients had restenosis of the airway, as revealed by laryngoscopic examination during six-month follow-up. Voice outcomes were measured by the patients by use of a $100 \mathrm{~mm}$ visual analog scale. Only one patient considered her voice socially unacceptable. This study is limited by the need for all patients to receive a tracheotomy at the time of surgery, and the need for an open approach.

\section{Dynamic Opening}

The ideal surgical technique would be one that improved glottic opening without having a detrimental effect on phonation or deglutition. A few groups have examined selective reinnervation of the PCA muscles, with some promising results [13, 14•, 15, 16]. Li et al. [14•] have published the most extensive data on selective PCA reinnervation for BVFI. In their procedure, the patient first received a tracheotomy, then underwent direct laryngoscopy to determine whether or not the CA joint was fixed. If the joints were immobile, they would proceed with endoscopic arytenoidectomy. Otherwise, an incision was made in the neck, and the carotid sheath contents in the left neck were retracted laterally to identify the phrenic nerve. The nerve was then transected at the level of the clavicle and set aside. The left RLN was then traced proximally until the site of injury was identified, and then distally as it began branching inside the larynx. All adductor branches of the RLN were transected, with care taken to avoid the abductor branches. The right RLN was then identified and its adductor branches were all transected. The phrenic nerve was transposed superiorly and anastamosed to the distal left RLN. A nerve jump graft using a portion of the great auricular nerve was then anastamosed from the left intralaryngeal adductor trunk of the RLN to the distal right RLN. The transected proximal adductor branches of the right RLN were then implanted into the right PCA muscle. 
In their study, 44 patients underwent selective reinnervation. Unilateral or bilateral vocal fold abduction was accomplished for 41 patients, but three later had deterioration. Of the remaining 38 patients, all were successfully decannulated 3-8 months after surgery. Grading of the voice by use of the GBRAS scale revealed no significant differences pre and post-surgery $(P>0.05$ for each value). Pulmonary function testing results, with the exception of maximum inspiratory pressure, returned to normal reference values within 12 months of surgery. Maximum inspiratory pressure for these patients at 12 months, while still significantly lower than the normal reference value, was still higher than the preoperative value $(P<0.05)$. Marie et al. [17] had previously published results for twelve patients for whom he had performed combined reinnervation (for both abductor and adductor muscle groups), but improvement was observed for only three of six evaluable patients. To date, this is the most successful reported study of selective PCA reinnervation. The results must be validated, and the surgery is very technically challenging.

Another approach to dynamic opening of the larynx is laryngeal pacing. Zealear et al. [18, 19] at Vanderbilt University have led the development of the Itrel II system. Reportedly, studies were discontinued because of problems with electrode design and materials. However, this group has been working on the development with a different type of device. One surgical concern with placement of electrodes for laryngeal pacing have been the risks of further surgical damage to the RLN and/or scarring that could limit laryngeal mobility, and thus hamper the effects of pacing. Förster et al. [20•] recently published results from a novel technique for electrode insertion in minipigs. It involved obtaining a direct laryngoscopic view of the pig larynx, and incising the anterior neck for access. A special trocar was then inserted submucosally toward the ventral cricoid lamina before then piercing the cartilage. Electrical signals then demonstrated its presence in the PCA. Monophasic pulses were delivered until a "sweet spot" was found with good abduction and very little activation of the adductors. The authors were able to successfully insert two functioning electrodes in 7 of 8 animals tested. The subjects tolerated the procedure well, with minimum laryngeal edema perioperatively. Although these results are indicative of the success of this innovative electrode placement, further studies are certainly warranted to assess electrode durability and the risk of migration.

\section{Conclusions}

In summary, treatment of BVFI continues to be a challenge. Preserving voice while improving airway is difficult, and at times, impossible. Thorough patient counseling is necessary before surgical steps that will result in permanent deterioration of one's ability to communicate. Static surgery techniques have remained almost unchanged in recent years. Although the papers reviewed here indicate recent modifications are viable, there is yet no clear consensus on the best technique.

\section{Compliance with Ethics Guidelines}

Conflict of Interest Joseph P. Bradley and Adam M. Klein declare no conflicts of interest.

Human and Animal Rights and Informed Consent This article does not contain any studies with human or animal subjects performed by the authors.

\section{References}

Papers of particular interest, published recently, have been highlighted as:

- Of importance

•- Of major importance

1. - Sapundzhiev N, Lichtenberger G, Eckel HE, Friedrich G, Zenev I, Toohill RJ, et al. Surgery of adult bilateral vocal fold paralysis in adduction: history and trends. Eur Arch Otorhinolaryngol. 2008;265:1501-14. Excellent, comprehensive review about the treatment of bilateral paralysis over the past century.

2. Halum SL, Ting JY, Plowman EK, Belafsky PC, Harbarger CF, Postma GN, et al. A multi-institutional analysis of tracheotomy complications. Laryngoscope. 2012;122:38-45.

3. Shah RK, Lander L, Berry JG, Nussenbaum B, Merati A, Roberson DW. Tracheotomy outcomes and complications: a national perspective. Laryngoscope. 2012;122:25-9.

4. Gilony D, Gilboa D, Blumstein T, Murad H, Talmi YP, Kronenberg $\mathrm{J}$, et al. Effects of tracheostomy on well-being and bodyimage perceptions. Otolaryngol Head Neck Surg. 2005;133: 366-71.

5. Ossoff RH, Sisson GA, Duncavage JA, Moselle HI, Andrews PE, McMillan WG. Endoscopic laser arytenoidectomy for the treatment of bilateral vocal cord paralysis. Laryngoscope. 1984;94:1293-7.

6. Dennis DP, Kashima H. Carbon dioxide laser posterior cordectomy for treatment of bilateral vocal cord paralysis. Ann Otol Rhinol Laryngol. 1989;98:930-4.

7. - Yilmaz T. Endoscopic total arytenoidectomy for bilateral abductor vocal fold paralysis: a new flap technique and personal experience with 50 cases. Laryngoscope. 2012;122:2219-26. Modification made to total arytenoidectomy procedure to help limit granulation \& scarring and maintain tension on the vocal fold.

8. - Semmler M, Keck T, Reiter R, Gruen PM. Endolaryngeal posterior mucosal flap for surgical repair of posterior glottic stenosis. Auris Nasus Larynx. 2011;38:608-11. New novel mucosal advancement flap to cover the defect from resection of early stage posterior glottic stenosis.

9. Lichtenberger G. Reversible immediate and definitive lateralization of paralyzed vocal cords. Eur Arch Otorhinolaryngol. 1999;256:407-11. 
10. - Katilmis H, Ozturkcan S, Basoglu S, Aslan H, Ilknur AE, Erdoğan NK, et al. New technique for the treatment of bilateral vocal cord paralysis: Vocal and ventricular fold lateralization using crossing sutures with thyroplasty technique. Acta Otolaryngol. 2011;131:303-9. Modification to the vocal fold lateralization technique in order to help prevent suture breakage and the need for revision.

11. - Songu M, Aslan H, Denizoglu I, Ozkul Y, Basoglu S, Ates D, et al. Vocal and ventricular fold lateralization using crossing sutures with the thyroplasty window technique for bilateral vocal fold immobility: long-term results. Acta Otolaryngol. 2013;133:1201-6. Follow-up study to Katilmis et al 2011 looking at the long-term results of the crossed suture vocal fold lateralization.

12. • Yagudin RK, Demenkov VR, Yagudin KF. Plastic cordotomy in the treatment of bilateral vocal fold immobility. Head Neck. 2012;34:1753-8. Open technique developed to change vocal fold positioning without destroying the substance of the vocal fold.

13. Crumley RL. Phrenic nerve graft for bilateral vocal cord paralysis. Laryngoscope. 1983;93:425-8.

14. - Li M, Chen S, Zheng H, Chen D, Zhu M, Wang W, et al. Reinnervation of bilateral posterior cricoarytenoid muscles using the left phrenic nerve in patients with bilateral vocal fold paralysis. PLoS One. 2013;8:e77233. Largest study published to date examining selective reinnervation for bilateral paralysis.
15. Zheng H, Zhou S, Li Z, Chen S, Zhang S, Huang Y, et al. Reinnervation of the posterior cricoarytenoid muscle by the phrenic nerve for bilateral vocal cord paralysis in humans. Zhonghua Er Bi Yan Hou Ke Za Zhi. 2002;37:210-4.

16. Marie JP, Lacoume Y, Magnier P, Dehesdin D. Selective bilateral motor reinnvervatin of the canine larynx. Laryngorhinootologie. 2000;79:S188-9.

17. Marie JP. Nerve reconstruction. In: Remacle M, Eckel HE, editors. Surgery of Larynx and Trachea. Heidelberg: Springer; 2010. p. 279-94.

18. Zealear DL, Billante CR, Courey MS, Netterville JL, Paniello $\mathrm{RC}$, Sanders I, et al. Reanimation of the paralyzed human larynx with an implantable electrical stimulation device. Laryngoscope. 2003;113:1149-56.

19. Zealear DL, Swelstad MR, Sant'Anna GD, Bannister RA, Billante CR, Rodriguez RJ, et al. Determination of the optimal conditions for laryngeal pacing with the Itrel II implantable stimulator. Otolaryngol Head Neck Surg. 2001;125:183-92.

20. • Förster G, Arnold D, Bischoff SJ, Schubert H, Scholle H-C, Müller AH. Laryngeal pacing in minipigs: in vivo test of a new minimal invasive transcricoidal electrode insertion method for functional electrical stimulation of the PCA. Eur Arch Otorhinolaryngol. 2013;270:225-31. Technique for minimally-invasive way of placing electrodes for laryngeal pacing of the PCA muscles. 